\title{
Drug Resistance Patterns in HIV Patients with Virologic Failure in Iran
}

\author{
Seyed Ahmad Seyed Alinaghi ${ }^{1}$, Mehrnaz Rasoolinejad ${ }^{1}$, Zeinab Najafi ${ }^{1}$, Omid Dadras ${ }^{2}$, Ehsan \\ Malekianzadeh ${ }^{3,{ }^{*}}$ and Ali Mirzazadeh ${ }^{4,5}$ \\ ${ }^{1}$ Iranian Research Center for HIV/AIDS, Iranian Institute for Reduction of High-Risk Behaviors, Tehran University of Medical Sciences, Tehran, Iran \\ ${ }^{2}$ Department of Global Health and Socioepidemiology, Graduate School of Medicine, Kyoto University, Kyoto, Japan \\ ${ }^{3}$ Dezful University of Medical Sciences, Dezful, Iran \\ ${ }^{4}$ HIV/STI Surveillance Research Center, and WHO Collaborating Center for HIV Surveillance, Kerman University of Medical Sciences, Kerman, Iran \\ ${ }^{5}$ Department of Epidemiology and Biostatistics, Institute for Global Health Sciences, University of California San Francisco, CA, USA \\ Corresponding author: Dezful University of Medical Sciences, Dezful, Iran. Email: ehsanmalekianzadeh@gmail.com
}

Received 2019 July 21; Revised 2019 December 13; Accepted 2019 December 15.

\begin{abstract}
We reviewed the medical charts of 1,700 patients diagnosed with HIV who referred to a central HIV clinic in Tehran between 2004 and 2017. Participants who had a viral load of $>200$ copies/mL after six months or more on antiretroviral therapy (ART) were grouped as virologic failure $(\mathrm{VF})$. We assessed the demographic characteristics, diagnosis date, first ART regimen, and resistance to various ART drugs. Out of 1,700 patients, $72(4.2 \%)$ had a treatment failure. Among those with treatment failure, $51.3 \%$ were on zidovudine + lamivudine + efavirenz, 18.1\% were on tenofovir + lamivudine + lopinavir/ritonavir, and $12.5 \%$ were on tenofovir + emtricitabine + efavirenz. In patients with treatment failure, the highest resistance was to nucleoside reverse transcriptase inhibitors (NRTIs) and non-nucleoside reverse transcriptase inhibitors (NNRTIs) combination (44.4\%). In these patients, resistance to tenofovir (one of the NRTIs) was $29.1 \%$. The highest treatment failure was observed among patients treated with nevirapine (NVP) and efavirenz (EFV)-based regimen. Our findings suggest that protease inhibitors should be considered as first-line drugs in ART regimens in VF patients in Iran.
\end{abstract}

Keywords: HIV/AIDS, Virologic Failure, Antiretroviral Therapy, Iran

\section{Background}

In 2017, an estimated 36.9 million people were living with HIV worldwide. Among them, 21.7 million were receiving antiretroviral therapy (ART) and 0.94 (0.67-1.3) million died from AIDS-related illnesses (1). The HIV pandemic is still a public health issue worldwide, especially in developing countries $(2,3)$. The disease is also growing in East Mediterranean region countries, including Iran $(4,5)$. According to the national HIV registry system, in 2017, the number of people living with HIV (PLWH) was 34,949 in Iran, including $84 \%$ men and $16 \%$ women, and the number of AIDS-related deaths was 9,477 by March $2017(2,6)$.

Since 2008, over eight million people received ART in developing countries (7). The ART regimens could significantly increase the quality and quantity of life of PLWH. An appropriate treatment, chosen at the right time, is crucial to achieving favorable outcomes. With effective treatment, there could be a substantial reduction in viral load and an increase in the number of CD4 cells; however, about $12 \%$ to $32 \%$ of patients fail to achieve these desirable outcomes (8, 9).
Besides the ART expansion, an emerging problem is HIV drug resistance (HIVDR) mutants, which are attributed to HIV mutating and replicating capabilities in the presence of ART drugs. The HIVDR deactivates the drugs which formerly could control viral replication. This has led to attempts in introducing more effective medications in ART regimens, which carry new side effects and consequently impose a more economic burden on both patients and health systems $(7,10)$.

Treatment failure and further spread of HIVDR mutants compromise the effectiveness of ART to meet "the last 90" target for viral suppression. It also increases HIV mortality and morbidity $(7,10,11)$. Therefore, appropriate surveillance of HIV patients receiving ART should be implemented to improve adherence, which is essential to achieve the desired outcome and prevent the emergence of HIVDR mutants (7).

In Iran, ART is available and free of charge for all PLWH. Despite its beneficial therapeutic effects, recently, a growing body of evidence has shown that the first-line treatment fails sometimes; however, it is not clear which drug fails and how frequent it occurs $(4,10)$. Early detection of 
treatment failure could substantially reduce the complications and prevent new viral mutants to emerge.

\section{Objectives}

Therefore, in this study, we aimed to investigate the prevalence of treatment failure and patterns of resistance to various ART drugs among PLWH in a major referral hospital in Iran.

\section{Methods}

\subsection{Participants}

We reviewed the medical charts of 1,700 HIV patients who referred to the voluntary counseling and testing(VCT) center of Imam Khomeini Hospital in Tehran between 2004 and 2017. Patients who lost to care, died, transferred out, or incarcerated were excluded. We found a total of 72 patients with virologic failure $(\mathrm{VF})$.

\subsection{Instruments}

The patient's demographic characteristics, viral load markers, TCD4+ count, and selected ART regimen were extracted from the patients' medical records using an information datasheet. The history of treatment failure, drug resistance results, and alternative regimens were also recorded. Treatment failure was determined based on the virologic features of patients. The 2018 AIDS info instruction (retrieved from https://aidsinfo.nih.gov/guidelines) was applied to determine treatment failure. Based on this instruction, the presence of 200 or more copies per milliliter of viral load after six months of continuous, effective ART was considered a treatment failure. Resistance tests were performed for the viral loads of more than 1,000 copies/mL and based on the results, the second line regimen began. We included all drug resistance mutations that presented low-, intermediate-, or high-level resistance. The RNA genome of HIV has several genes such as reverse transcriptase (RT), protease (Pro), and integrase (INT). These genes may mutate and cause drug resistance in HIV-positive patients. For this reason, drug resistance was evaluated by the amplification of these genes using specific primers by polymerase chain reaction (PCR) and sequencing of products. Then, the results of the analysis were extracted based on the Stanford HIV drug resistance website (hivdb.stanford.edu).

\subsection{Ethical Considerations}

The study protocol was approved by the Institutional Review Board (IRB) of Tehran University of Medical Sciences (TUMS). All the information sheets were secured in a locked shelve where only authorized persons had access to. The digital data file was secured by a password that was only known to study researchers.

\subsection{Statistical Analysis}

To analyze the data, we used SPSS software (version 22). Descriptive statistics were used to describe the study participants and frequency of different drug resistance patterns.

\section{Results and Discussion}

\subsection{Study Population}

We analyzed the data of 72 patients with VF. The majority of patients were male (68.1\%), aged 35 - 50 years (61.1\%), and unemployed (36.1\%). Moreover, they mostly had high school education (51.4\%), a history of drug use (55.6\%), and acquired infection from high-risk heterosexual contacts (54.2\%). Most of the patients were on an ART regimen for 37 months or longer (86.1\%) (Table 1$)$.

\subsection{Virologic Failure}

The mean value of the first CD4 count (CD4 count right after ART initiation) was 262.4 cells $/ \mu$ L and the mean value of the last CD4 count (CD4 count after ART) was 349.6 cells/ $\mu \mathrm{L}$. The mean value of the first viral load was 191,309.7 copies/mL while the mean value of the last viral load was 32,312.2 copies/mL. Following treatment, the mean value of the last CD4 count was significantly higher than the mean value of the first $\mathrm{CD} 4$ count $(\mathrm{P}<0.001)$. In total, 72 out of 1700 (4.2\%) HIV patients experienced treatment failure. The highest numbers of VF patients were receiving NNRTIs ( $\mathrm{n}=$ $19 ; 26.4 \%)$ and NNRTIs + NRTIs ( $\mathrm{n}=32 ; 44.4 \%)$.

The most frequent failed regimens were AZT-3TC-EFV (51.3\%), TDF-3TC-LPV/r (18.1\%), and TDF-FTC-EFV (12.5\%) (Table $2)$. The highest resistance levels were related to nevirapine (NVP) (73.6\%) and efavirenz (EFV) (70.8\%) (Table 3). There was no significant relationship between drug type and resistance level (P value > 0.05).

We found that HIV treatment failed in less than $5 \%$ of patients. The highest drug resistance belonged to NRTIs and NNRTIs combination. The highest rate of VF was attributed to nevirapine (NVP) and efavirenz (EFV). Resistance to tenofovir (TDF), as the most commonly prescribed NRTIs, was observed in less than one-third of patients.

Offering HIV mediations to all patients at the time of diagnosis or at early as possible after diagnosis worldwide 


\begin{tabular}{|c|c|}
\hline Characteristics & Values $^{\mathbf{b}}$ \\
\hline Male & $49(68.1)$ \\
\hline \multicolumn{2}{|l|}{ Age group, y } \\
\hline $16-25$ & $1(1.4)$ \\
\hline $26-34$ & $17(23.6)$ \\
\hline $35-50$ & $45(61.1)$ \\
\hline$>50$ & $8(11.1)$ \\
\hline Unemployed & $26(36.1)$ \\
\hline \multicolumn{2}{|l|}{ Education level } \\
\hline Illiterate & $3(4.2)$ \\
\hline Elementary school & $17(23.6)$ \\
\hline High school & $37(51.4)$ \\
\hline Academic & $9(12.5)$ \\
\hline \multicolumn{2}{|l|}{ Marital status } \\
\hline Single & $29(40.3)$ \\
\hline Married & $29(40.3)$ \\
\hline Divorced & $6(8.3)$ \\
\hline Widow & $3(4.2)$ \\
\hline \multicolumn{2}{|l|}{ Smoking status } \\
\hline Ex-smoker & $11(15.3)$ \\
\hline Current smoker & $35(48.6)$ \\
\hline Past history of drug use & $40(55.6)$ \\
\hline \multicolumn{2}{|l|}{ Transmission route } \\
\hline Heterosexual & $39(54.2)$ \\
\hline Homosexual & $3(4.2)$ \\
\hline Drug injection & $22(30.6)$ \\
\hline $\begin{array}{l}\text { Others (mother to child, blood/blood } \\
\text { product transfusion) }\end{array}$ & $4(5.6)$ \\
\hline \multicolumn{2}{|l|}{ ART duration, month } \\
\hline $0-12$ & $4(5.6)$ \\
\hline $13-24$ & $3(4.2)$ \\
\hline $25-36$ & $2(2.8)$ \\
\hline$\geq 37$ & $62(86.1)$ \\
\hline
\end{tabular}

${ }^{\mathrm{a}}$ Values are expressed as No. (\%).

${ }^{\mathrm{b}}$ Subgroups do not always add up to total due to missing data.

raises concerns about emerging drug resistance, mainly due to the lack of compliance in regular and continuous drug intake. The patient's adherence to therapy is a key component of a successful ART program $(12,13)$. Lack of knowledge in patients about ART and the importance of adherence, misconception about side effects, and dealing with many other priorities in life (14), feeling sick (15), and

\begin{tabular}{ll}
$\begin{array}{l}\text { Table 2. Antiretroviral Therapy Regimens for People Living with HIV experiencing } \\
\text { Treatment Failure, Tehran, Iran, } 2004-2017^{\mathrm{a}}\end{array}$ \\
\hline Regimen & Values \\
\hline TDF-FTC-EFV & $9(12.5)$ \\
\hline AZT-3TC-EFV & $37(51.3)$ \\
\hline TDF-3TC-LPV/r & $13(18.1)$ \\
\hline TDF-3TC-EFV & $7(9.7)$ \\
\hline TDF-FTC-LPV/r & $7(10)$ \\
\hline TDF-3TC-ATV/r & $6(8.4)$ \\
\hline AZT-3TC-NVP & $6(8.4)$ \\
\hline AZT-3TC-LPV/r & $5(7)$ \\
\hline TDF-FTC-ATV/r & $4(5.6)$ \\
\hline
\end{tabular}

${ }^{\mathrm{a}}$ Values are expressed as No. (\%).

other co-infections and concomitant diseases (16) were reported as factors associated with ART nonadherence.

An increasing concern besides the ART extension is the emerging of HIVDR mutants, which is attributed to HIV mutating and replicating capabilities in the presence of ART drugs (8). In the current study, the highest resistance was observed to NRTIs + NNRTIs regimens (44\%). In fact, the NNRTIs regimens sustained the most frequent drug resistance among which, NVP (74\%) and EFV (71\%) demonstrated the highest resistance rates. A similar study in Iran found resistance to NVP (21.3\%) and EFV (19.7\%) as the most frequent drug resistance rates among HIV patients (17). They also found that $36.1 \%$ of HIV patients had at least one mutation related to RT inhibitors. The RT inhibitor mutations were also reported to reduce the susceptibility to NRTIs and NNRTIs $(17,18)$. The G190A mutation is another common mutation that occurs in $36 \%$ of cases $(19,20)$ and it could reduce the viral response to NVP and EFV by more than 50 and 5 - 10 times, respectively $(21,22)$. There are also other mutations $(23,24)$ that can cause HIVDR.

Our study had three major limitations. First, we could not locate the data for all patients, particularly children, which limited the study external validity. Second, we used the medical record data and thus, the completeness and quality of data were not perfect and varied over time. Lastly, we assessed the drug resistance patterns only among patients with treatment failure.

Despite the limitations, our study characterized the ART resistance patterns among HIV patients for whom HIV treatment failed. Our findings propose to use protease inhibitors as first-line drugs in ART regimens in patients with VF. 


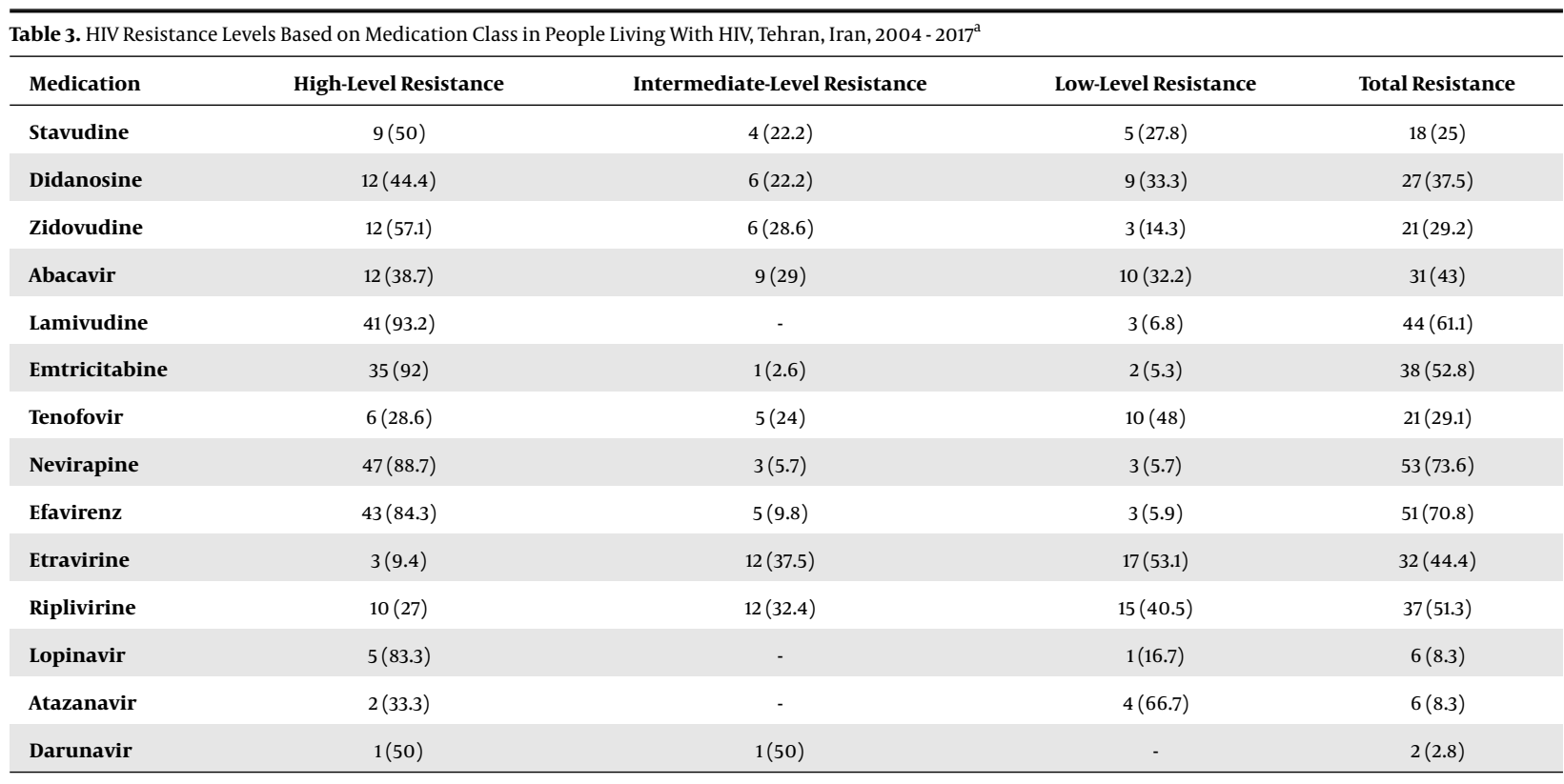

${ }^{\mathrm{a}}$ Values are expressed as No. (\%).

\section{Supplementary Material}

Supplementary material(s) is available here [To read supplementary materials, please refer to the journal website and open PDF/HTML].

\section{Acknowledgments}

We thank the staff in the VCT center for their help.

\section{Footnotes}

Authors' Contribution: Seyed Ahmad Seyed Alinagh designed and performed experiments. Mehrnaz Rasoolinejad performed experiments. Zeinab Najafi designed experiments and co-wrote the paper. Omid Dadras performed bioinformatic analyses. Ehsan Malekianzadeh performed experiments. Ali Mirzazadeh supervised research.

Conflict of Interests: The authors declare that they have no conflict of interest.

Ethical Approval: The study was approved by the Tehran University of Medical Sciences (TUMS) Ethics Committee (reference number: IR.TUMS.VCR.REC.1397.966).

Funding/Support: This study was also supported by the National Institutes of Health (code: R25 MH064712).

\section{References}

1. People living with HIV receiving art. 2019, [cited 2019 Jun 30]. Available from: https://aidsinfo.unaids.org/.
2. Rahmati-Najarkolaei F, Niknami S, Aminshokravi F, Bazargan M, Ahmadi F, Hadjizadeh E, et al. Experiences of stigma in healthcare settings among adults living with HIV in the Islamic Republic of Iran. J Int AIDS Soc. 2010;13:27. doi: 10.1186/1758-2652-13-27. [PubMed: 20649967]. [PubMed Central: PMC2919446].

3. Alinaghi SA, Baesi K, Hosseini SY, Teimoori A, Gholami M. Diversity and Global Epidemiology of HIV. UAE: Bentham Science; 2016.

4. Sardashti S, Samaei M, Firouzeh MM, Mirshahvalad SA, Pahlaviani FG SeyedAlinaghi S. Early initiation of antiretroviral treatment: Challenges in the Middle East and North Africa. World J Virol. 2015;4(2):13441. doi: 10.5501/wjv.v4.i2.134. [PubMed: 25964878]. [PubMed Central: PMC4419117].

5. Fahimfar N, Sedaghat A, Hatami H, Kamali K, Gooya M. Counseling and harm reduction centers for vulnerable women to HIV/AIDS in Iran. Iran J Public Health. 2013;42(Supple1):98-104. [PubMed: 23865025]. [PubMed Central: PMC3712587].

6. Joulaei H, Motazedian N. Primary Health Care Strategic key to control HIV|AIDS in Iran. Iran J Public Health. 2013;42(5):540-1. [PubMed: 23802115]. [PubMed Central: PMC3684466].

7. Seyed Alinaghi SA. Current studies in HIV research. 2. United Arab Emirates: Bentham Science Publishers; 2006. p. 99-180.

8. Seyed Alinaghi SA, Farhoudi B, Mohraz M, Alipour A, Golrokhy R, Hosseini $\mathrm{M}$, et al. Adherence to antiretroviral therapy and tuberculosis treatment in a prison of Tehran, Iran. Infect Disord Drug Targets. 2016;16(3):199-203. doi: 10.2174/1871526516666160616111308. [PubMed: 27311561].

9. Baesi K, Ravanshad M, Ghanbarisafari M, Saberfar E, Seyedalinaghi S, Volk JE. Antiretroviral drug resistance among antiretroviral-naive and treatment experienced patients infected with HIV in Iran. J Med Virol. 2014;86(7):1093-8. doi: 10.1002/jmv.23898. [PubMed: 24740443].

10. Baesi K, Moallemi S, Farrokhi M, Alinaghi SA, Truong HM. Subtype classification of Iranian HIV-1 sequences registered in the HIV databases, 2006-2013. PLoS One. 2014;9(9). e105098. doi: 10.1371/journal.pone.0105098. [PubMed: 25188443]. [PubMed Central: PMC4154867]. 
11. Paydary K. Emerging HIV drug resistance in the resource-poor world: Challenges and strategies. J AIDS Clin Res. 2013;1(S5). doi: 10.4172/21556113.s5-006.

12. Langebeek N, Gisolf EH, Reiss P, Vervoort SC, Hafsteinsdottir TB, Richter $\mathrm{C}$, et al. Predictors and correlates of adherence to combination antiretroviral therapy (ART) for chronic HIV infection: A meta-analysis. BMC Med. 2014;12:142. doi: 10.1186/PREACCEPT1453408941291432. [PubMed: 25145556]. [PubMed Central: PMC4148019].

13. Bock P, James A, Nikuze A, Peton N, Sabapathy K, Mills E, et al. Baseline CD4 count and adherence to antiretroviral therapy: A systematic review and meta-analysis. J Acquir Immune Defic Syndr. 2016;73(5):514-21. doi: 10.1097/QAI.0000000000001092. [PubMed: 27851712].

14. Mills EJ, Nachega JB, Bangsberg DR, Singh S, Rachlis B, Wu P, et al. Adherence to HAART: A systematic review of developed and developing nation patient-reported barriers and facilitators. PLoS Med. 2006;3(11). e438. doi: 10.1371/journal.pmed.0030438. [PubMed: 17121449]. [PubMed Central: PMC1637123].

15. Saberi P, Neilands TB, Vittinghoff E, Johnson MO, Chesney M, Cohn SE. Barriers to antiretroviral therapy adherence and plasma HIV RNA suppression among AIDS clinical trials group study participants. AIDS Patient Care STDS. 2015;29(3):111-6. doi: 10.1089/apc.2014.0255. [PubMed 25615029]. [PubMed Central: PMC4352694].

16. Nachega JB, Parienti JJ, Uthman OA, Gross R, Dowdy DW, Sax PE, et al. Lower pill burden and once-daily antiretroviral treatment regimens for HIV infection: A meta-analysis of randomized controlled trials Clin Infect Dis. 2014;58(9):1297-307. doi: 10.1093/cid/ciu046. [PubMed 24457345]. [PubMed Central: PMC3982838].

17. Golmohammadi R, Baesi K, Moradi A, Farrokhi M, McFarland W, Parsamajd S. The first characterization of HIV-1 subtypes and drug resistance mutations among antiretrovirally treated patients in Kermanshah, Iran. Intervirology. 2017;60(1-2):33-7. doi: 10.1159/000478701. [PubMed: 28795954]

18. Gholami M, Sadeghi L, Baesi K, Rouzbahani N, Mohraz M. Survey of antiretroviral drug resistance pattern among HIV-infected patients with treatment failure in Iran. J Hum Virol Retrovir. 2015;3(1):1-6.

19. Bacheler LT, Anton ED, Kudish P, Baker D, Bunville J, Krakowski K, et al. Human immunodeficiency virus type 1 mutations selected in patients failing efavirenz combination therapy. Antimicrob Agents Chemother. 2000;44(9):2475-84. doi: 10.1128/aac.44.9.2475-2484.2000. [PubMed: 10952598]. [PubMed Central: PMC90088].

20. Reuman EC, Rhee SY, Holmes SP, Shafer RW. Constrained patterns of covariation and clustering of HIV-1 non-nucleoside reverse transcriptase inhibitor resistance mutations. J Antimicrob Chemother. 2010;65(7):1477-85. doi: 10.1093/jac/dkq140. [PubMed: 20462946]. [PubMed Central: PMC2882873].

21. Rhee SY, Liu T, Ravela J, Gonzales MJ, Shafer RW. Distribution of human immunodeficiency virus type 1 protease and reverse transcriptase mutation patterns in 4,183 persons undergoing genotypic resistance testing. Antimicrob Agents Chemother. 2004;48(8):3122-6. doi: 10.1128/AAC.48.8.3122-3126.2004. [PubMed: 15273130]. [PubMed Central: PMC478552].

22. Zhang Z, Xu W, Koh YH, Shim JH, Girardet JL, Yeh LT, et al. A novel nonnucleoside analogue that inhibits human immunodeficiency virus type 1 isolates resistant to current nonnucleoside reverse transcriptase inhibitors. Antimicrob Agents Chemother. 2007;51(2):42937. doi: 10.1128/AAC.01032-06. [PubMed: 17116677]. [PubMed Central: PMC1797749].

23. Samuel R, Julian MN, Paredes R, Parboosing R, Moodley P, Singh $\mathrm{L}$, et al. HIV-1 drug resistance by ultra-deep sequencing following short course zidovudine, single-dose nevirapine, and singledose tenofovir with emtricitabine for prevention of mother-tochild transmission. J Acquir Immune Defic Syndr. 2016;73(4):384-9. doi: 10.1097/QAI.0000000000001116. [PubMed: 27327263]. [PubMed Central: PMC5172515].

24. Naziri H, Baesi K, Moradi A, Aghasadeghi MR, Tabarraei A, McFarland $\mathrm{W}$, et al. Antiretroviral drug resistance mutations in naive and experienced patients in Shiraz, Iran, 2014. Arch Virol.2016;161(9):2503-9. doi: 10.1007/s00705-016-2955-z. [PubMed: 27368990]. 